\title{
High-Tensile Strength Tapes Show Greater Ultimate Failure Load and Less Stiffness Than High-Tensile Strength Sutures in a Subpectoral Biceps Tenodesis Porcine Model
}

\author{
Chih-Kai Hong, M.D., Wei-Ren Su, M.D., M.Sc., Fa-Chuan Kuan, M.D., \\ Yueh Chen, M.D., M.Sc., Chen-Hao Chiang, M.D., and Kai-Lan Hsu, M.D.
}

Purpose: To compare the biomechanical properties of high-tensile strength tape and a high-tensile strength suture in subpectoral biceps tenodesis using a suture anchor in a porcine tendon model. Methods: A total of 24 artificial composite (polymer and glass fiber) humeri and porcine flexor profundus tendons were used. Two types of suture materials, hightensile strength sutures (group S) and high-tensile strength tapes (group T), were evaluated. After we inserted metallic suture anchors with either 2 sutures or tapes $5 \mathrm{~cm}$ from the superomedial corner of the greater tuberosity, a Krackow suture technique was used to secure the tendons. After a preload of $5 \mathrm{~N}$ for 2 minutes, a cyclic loading test from 5 to $70 \mathrm{~N}$ was conducted for 500 cycles. Finally, the specimen was loaded to failure at a rate of $1 \mathrm{~mm} / \mathrm{s}$. Results: There were no significant between-group differences in elongation after cyclic loading and elongation at failure load for group $\mathrm{S}$ and group T $(P=.977$ and .630 , respectively). The ultimate failure loads in group T $(278.2 \pm 54 \mathrm{~N})$ were significantly greater than those in group $\mathrm{S}(249.4 \pm 32 \mathrm{~N})(P=.028)$. In contrast, the stiffness values in group $\mathrm{T}(28.5 \pm 4.0 \mathrm{~N} / \mathrm{mm})$ were significantly lower than those in group $\mathrm{S}(32.3 \pm 4.5 \mathrm{~N})(P=.028)$. Ten specimens in group $\mathrm{S}$ and 8 specimens in group $\mathrm{T}$ failed, with tendons being cut through by the sutures, whereas the other 2 specimens in group $S$ and 4 specimens in group $\mathrm{T}$ failed due to suture breakage. Conclusions: Using high-tensile strength tapes in subpectoral biceps tenodesis using a suture anchor leads to significantly greater ultimate failure load as compared with using high-tensile strength sutures in a porcine model. Although lower levels of stiffness were found in high-tensile strength tape group, the difference in the means were not large between 2 groups. Clinical Relevance: A strong suture-tendon structure may prevent clinical failure of a subpectoral biceps tenodesis using a suture anchor.

$\mathbf{L}$ esions of the long head of the biceps brachii (LHB) tendon can possibly lead to anterior shoulder pain and shoulder dysfunction. ${ }^{1,2}$ Both biceps tenotomy and tenodesis are considered to be effective treatments for symptomatic LHB pathology. ${ }^{3-7}$ Recently, biceps tenodesis has become popular, ${ }^{8}$ and members of the American Shoulder and Elbow Society seem to favor biceps tenodesis over tenotomy. ${ }^{9}$ Among various tenodesis techniques, open subpectoral biceps tenodesis remains a well-liked option because it features several advantages, including simplicity, direct visualization, and prevention of length-tension relation mismatch. ${ }^{10-12}$ Recently, some authors have promoted the use of suture anchors in biceps tenodesis since they have been found to result
From the Department of Orthopaedic Surgery (C.-K.H., W.-R.S., F.-C.K., K.-L.H.) and Skeleton Materials and Bio-compatibility Core Lab, Research Center of Clinical Medicine (W.-R.S.), National Cheng Kung University Hospital, College of Medicine, National Cheng Kung University, Tainan; Department of Biomedical Engineering (F.-C.K., K.-L.H.), National Cheng Kung University, Tainan; Department of Orthopaedic Surgery, Sin Lau Hospital, Tainan (Y.C.); and Department of Orthopaedic Surgery, Chiayi Christian Hospital, Chiayi (C.-H.C), Taiwan.

The authors report that they have no conflicts of interest in the authorship and publication of this article. Full ICMJE author disclosure forms are available for this article online, as supplementary material.

\footnotetext{
Received January 26, 2020; accepted July 8, 2020.

Address correspondence to Kai-Lan Hsu, M.D., Department of Orthopaedic Surgery, National Cheng Kung University Hospital, College of Medicine, National Cheng Kung University, No. 138, Sheng-Li Rd, Tainan City, Taiwan 70428.E-mail:dulendulen@gmail.com

(C) 2020 THE AUTHORS. Published by Elsevier Inc. on behalf of the Arthroscopy Association of North America. This is an open access article under the CC BY-NC-ND license (http://creativecommons.org/licenses/by-nc-nd/4.0/). 2666-061X/20122

https://doi.org/10.1016/j.asmr.2020.07.018
} 
in less anatomical failure ${ }^{13}$ and may reduce some possible complications, such as tendon damage or humeral fracture. ${ }^{10,14}$

The structure at the suture-tendon junction will greatly affect the biomechanical properties of biceps tenodesis when using a suture anchor. ${ }^{15-17}$ Previous biomechanical studies focused on the suture techniques for fixing these tendons using suture anchors and indicated that more complex suture techniques, such as the Krackow suture technique, may significantly increase the ultimate failure loads of the tenodesis constructs. $^{15-17}$ However, despite the use of the Krackow suture, the common failure mechanism of tenodesis using a suture anchor has remained failure at the tendon-suture interface, including tendon split by sutures or suture rupture. ${ }^{14,16}$

A high-tensile strength tape has the potential to enhance the tendon-suture interface in the biceps tenodesis structure using a suture anchor. This kind of high-tensile strength tape contains ultra-high molecular weight polyethylene over the entire tape without a central core, which was developed to avoid tendon cut through by solid core sutures. ${ }^{18}$ Leishman and Chudik ${ }^{18}$ reported that this high-tensile strength tape has greater knot security, ultimate load to failure, and tensile stiffness than a high-tensile strength round core suture.

Although the high-tensile strength tape has a greater ultimate failure load as compared with the high-tensile strength suture and could possibly lead to less tendon cut through, ${ }^{18}$ little is known about its performance in subpectoral biceps tenodesis using a suture anchor. The purpose of this study is to compare the biomechanical properties of high-tensile strength tape and a high-tensile strength suture in subpectoral biceps tenodesis using a suture anchor in a porcine tendon model. We hypothesized that the high-tensile strength tape would have significantly greater ultimate failure load and stiffness as compared with the high-tensile strength suture in subpectoral biceps tenodesis using a suture anchor.

\section{Methods}

The current study was granted an exemption from the institutional review board in a medical center. The model for tenodesis biomechanical testing was in accordance with a previous study. ${ }^{16}$

\section{Specimens}

Fourth-generation composite humeri (model \#3404; Pacific Research Laboratories, Vashon, WA) were used in this study because previous studies have suggested it to be a reliable substitute for cadaver specimens for the purpose of biomechanical testing. ${ }^{19,20}$ It has been reported that the fourth-generation composite humerus not only reproduces the biomechanical properties of human bone but also reduces the interspecimen variability that occurs with cadaveric bone ${ }^{19,20}$ Finally, a

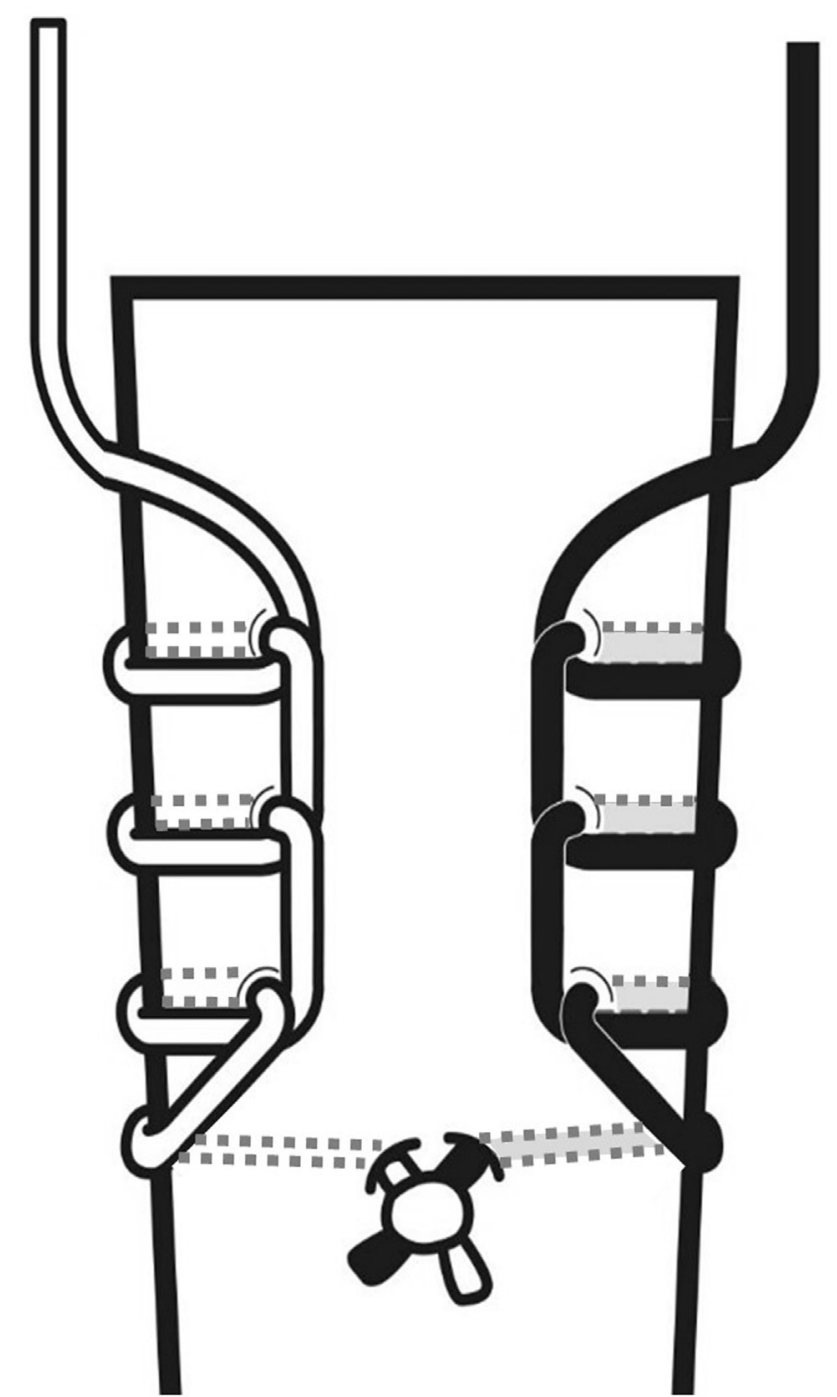

Fig 1. The illustration of Krackow suture technique from Arena and Dhawan. ${ }^{10}$

total of 24 large-size, left, fourth-generation composite humeri were used. The humerus was cut at mid-level of the shaft with a saw, and the proximal part of the humerus was preserved. The tenodesis site was located $5 \mathrm{~cm}$ distal from the superomedial corner of the greater tuberosity. ${ }^{16,21}$

A total of 24 porcine flexor foreleg tendons from fresh adult male porcine (mean age, 22 months) were chosen to simulate the LHB tendons because a previous study indicated that porcine flexor tendons have similar biomechanical properties to those of human cadaveric tendons. ${ }^{22}$ The trotters were stored at $-20^{\circ}$ and were thawed to room temperature before dissection. The quality of the tendon was assessed by a single author (C.-K.H.). Next, after removal of the attached soft tissue, the entire flexor tendon was harvested. A sizer was used for the preliminary selection to confirm the size of the tendon to be approximately $4.5 \mathrm{~mm}$, since the average diameter of LHB tendon has been reported to be 
Fig 2. Illustration of subpectoral biceps tenodesis constructs using suture anchors with (A) high-tensile strength sutures and (B) high-tensile strength tapes in left humerus composite bones.

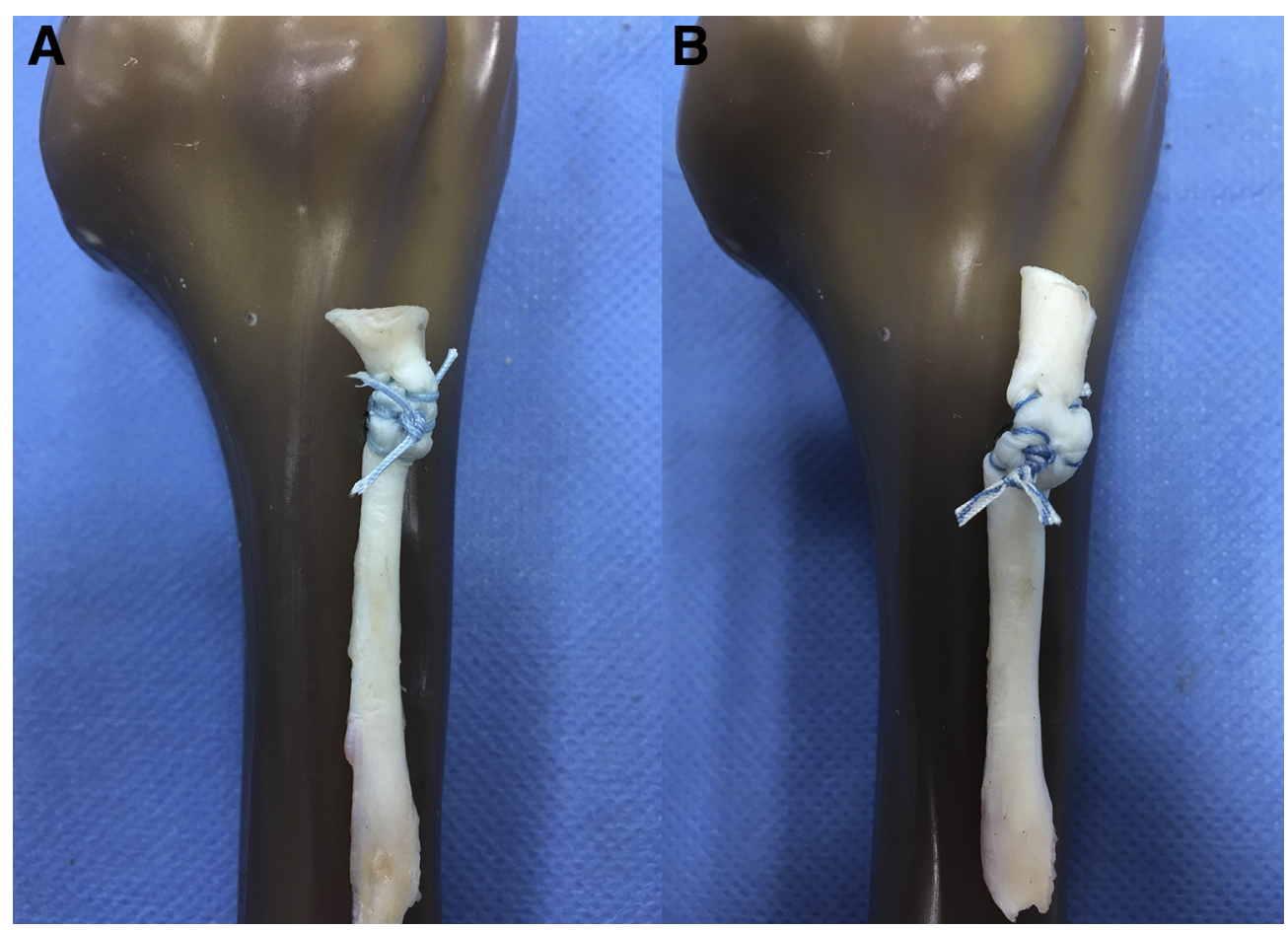

$4.4 \mathrm{~mm}$, ranging from 3.5 to $5.0 \mathrm{~mm} .^{23}$ Subsequently, the actual cross-sectional area of each tendon was measured with the use of a calibration scale, a digital camera, and image processing software (ImageJ, version 1.52p; National Institutes of Health, Bethesda, MD).

With a use of a random numbers table, the composite humeri and porcine flexor tendons were randomly divided into 2 groups: a high-tensile strength suture group (group S) and a high-tensile strength tape group (group T).

\section{High-Tensile Strength Suture Group (Group S)}

Metallic suture anchors (Corkscrew Suture Anchor, $5.0 \mathrm{~mm}$; Arthrex, Naples, FL) with double-loaded No. 2 high-tensile strength sutures (FiberWire; Arthrex) were used. The suture anchors were inserted at $90^{\circ}$ to the bone surface at the tenodesis site until the eyelets of the suture anchors were at level with the bone.

\section{High-Tensile Strength Tape Group (Group T)}

Metallic suture anchors (Corkscrew Suture Anchor, $5.0 \mathrm{~mm}$; Arthrex) were used for the tenodesis. Before the insertion of the anchor, the high-tensile strength sutures from the suture anchor were substituted with two high-tensile strength tapes (1.3-mm SutureTape; Arthrex). The suture anchors were inserted at $90^{\circ}$ to the bone surface at the tenodesis site until the eyelets of the suture anchors were level with the bone.

\section{Suture Technique}

The suture technique for LHB tendon fixation followed the method from Arena and Dhawan. ${ }^{10}$ Starting
$1.0 \mathrm{~cm}$ from the tendon end, 1 suture strand was used to run 3 locking stitches in a Krackow configuration on the lateral aspect of the tendon, whereas the other suture strand was sutured in the same fashion along the medial aspect of the tendon. Next, the 2 running sutures were tied together with half hitches (Fig 1). Then, the 2 remaining free ends of the sutures were passed through the tendon from the posterior-toanterior direction with one located at the proximal Krackow loop and the other at the distal Krackow loop. After these 2 suture ends are pulled, the tendon was attached to the tenodesis site on the humerus. Finally, the 2 sutures were tied together with 5 half hitches to secure the tendon on the tenodesis site (Fig 2).

\section{Testing Setup}

The prepared specimens were mounted in the material testing machine (AG-X; Shimadzu, Tokyo, Japan) for biomechanical testing (Fig 3). The humeral head of the composite bone was fixed via a customized jig connected to the base of the material testing machine. Another custom-made sinusoidal clamp, connected to the test actuator and an inline $1000-\mathrm{N}$ load cell, was used to secure the porcine tendon $10 \mathrm{~cm}$ from the tendon end. To ensure that the direction of the tensile force on the tendons was parallel to the longitudinal axis of the humerus, an X-Y table allowed the adjustment of the position of the construct.

The biomechanical testing protocol comprised pretensioning, cyclic loading, and load-to-failure tests. Each specimen was first preloaded to $5 \mathrm{~N}$ for 2 minutes. 


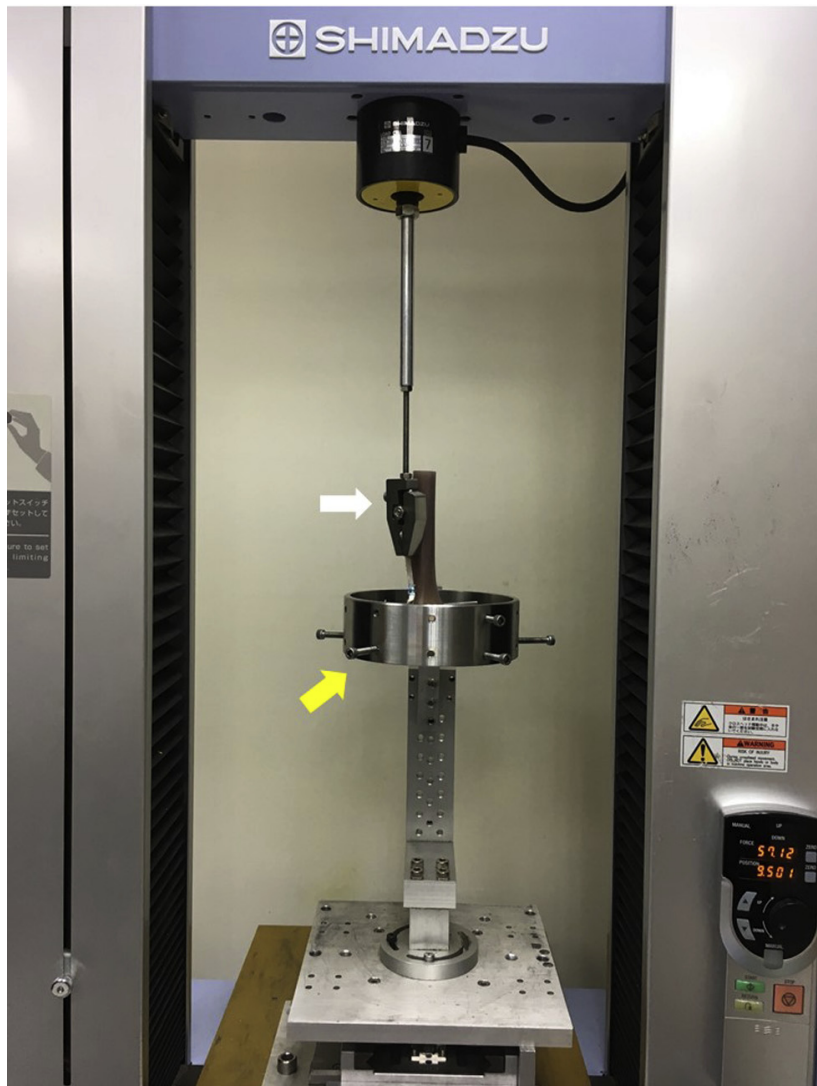

Fig 3. The experimental setup for biomechanical testing. The composite artificial humerus was mounted on the material testing machine with a customized securing device (yellow arrow), and the porcine flexor tendon was secured with a sinusoidal clamp (white arrow) which was connected to the load cell. Vertical cyclic and failure forces were applied.

Next, cyclic loading force from 5 to $70 \mathrm{~N}$ was given for 500 cycles. Finally, the specimen was loaded to failure at a constant crosshead rate of $1 \mathrm{~mm} / \mathrm{s}$. The aforementioned parameters were consistent with those in previous studies. ${ }^{14,16,24-27}$

During testing, the relevant parameters, including testing time, force, and actuator displacement, were recorded using Trapezium X software (version 1.00; Shimadzu, Tokyo, Japan). Displacement at cyclic loading could be acquired by calculating the difference between the actuator displacement in the first cycle at $5 \mathrm{~N}$ and that in the 500th cycle at $70 \mathrm{~N}$. The displacement at failure was calculated based on the peak displacement at the failure load and the initial preconditioned load at $5 \mathrm{~N}$ in the load-to-failure test. The yield point in the stress-strain curve was identified during load-to-failure test. The stiffness for the elastic region in the stress-strain curve calculated using the data of applied force and the displacement of whole construct. The failure mode of each specimen was recorded after the load-to-failure test.

\section{Statistical Analysis}

The required sample size for this biomechanical study was determined based on a pilot study with a total of 8 specimens randomly assigned to 2 groups (group $S$ and group $\mathrm{T}$ ) using $\mathrm{G}^{*}$ Power, version 3.1.3 software (Heinrich Heine-University of Dusseldorf, Dusseldorf, Germany). An $\alpha$ equal to 0.05 and a power $(1-\beta)$ of 0.80 were given to this pilot study model, and an effect size of 1.44 was obtained. Accordingly, a required sample size of 20 specimens was determined to be appropriate. We finally included 24 samples in total, with 12 in each group.

SPSS software for Windows (version 20.0; IBM Corp., Armonk, NY) was used for the statistical analysis. Descriptive statistics, including means and standard deviations, were obtained for both groups. The Mann-Whitney $U$ test was used to compare the ultimate failure load, stiffness, the elongation after cyclic loading, and elongation at failure load between the 2 groups. A $\chi^{2}$ test was used to compare the failure modes between 2 groups. The statistical significance was set as $P \leq .05$.

\section{Results}

The cross-sectional areas of the flexor tendons of the forelegs in group $\mathrm{S}\left(15.9 \pm 0.5 \mathrm{~mm}^{2}\right)$ and group $\mathrm{T}$ $\left(15.8 \pm 0.6 \mathrm{~mm}^{2}\right)$ were not significantly different $(P=.713)$. All specimens in groups $\mathrm{S}$ and $\mathrm{T}$ completed the cyclic loading tests as well as the load to failure tests.

The biomechanical testing results are concluded in Table 1. There were not significant between-group differences in the elongation after cyclic loading and elongation at failure load ( $P=.977$ and .630 , respectively).

Table 1. Results of Biomechanical Testing Between High-Tensile Strength Suture Group (Group S) and High-Tensile Strength Tape Group (Group T)

\begin{tabular}{lcc}
\hline Variables (Mean \pm SD) & Group S (n = 12) & Group T (n $=12)$ \\
\hline Cyclic elongation, mm & $5.9 \pm 1.4$ & $5.8 \pm 1.1$ \\
Failure elongation, mm & $10.9 \pm 2.5$ & $10.5 \pm 2.9$ \\
Ultimate failure load, N & $249.4 \pm 32$ & $278.2 \pm 54$ \\
Stiffness, N/mm & $32.3 \pm 4.5$ & $28.5 \pm 4.0$ \\
Failure mode & 10 tendon tears & 8 tendon tears \\
& 2 suture breakages & 4 suture breakages \\
\hline
\end{tabular}

SD, standard deviation.

*Significant between-group differences with the Mann-Whitney $U$ test. 

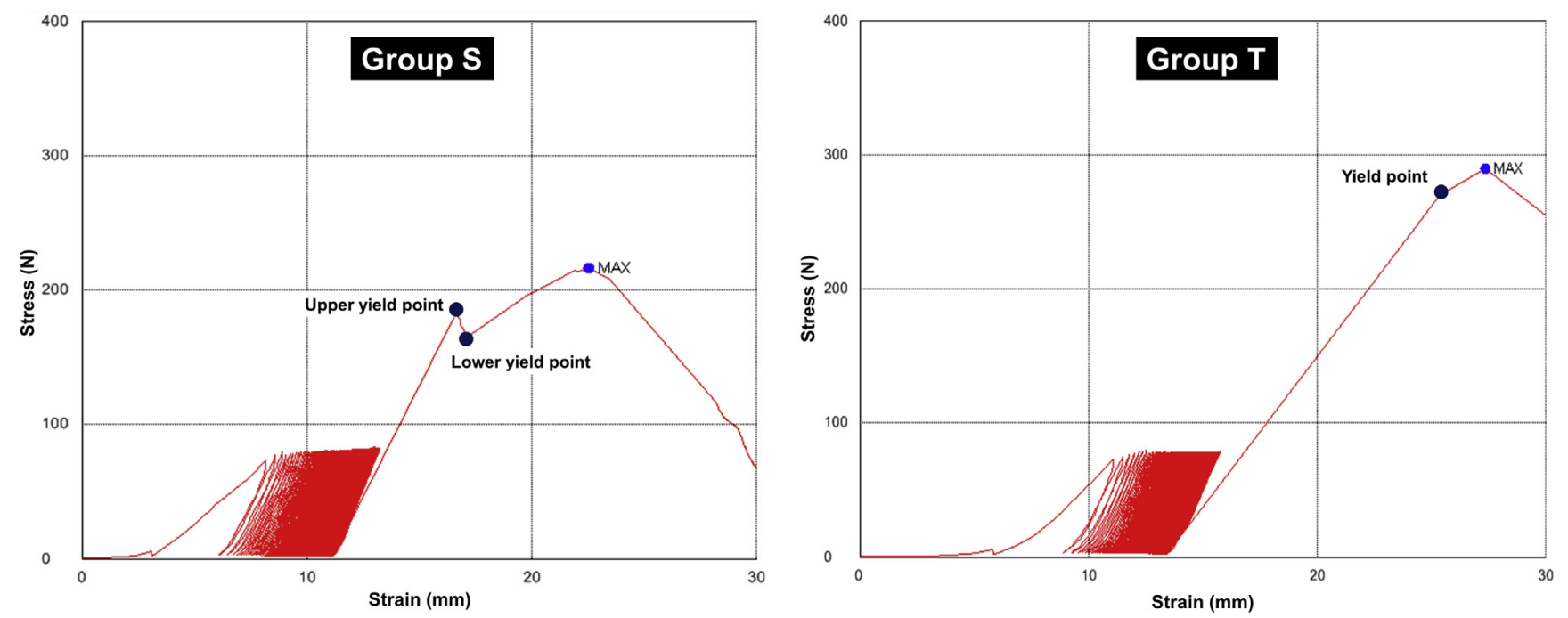

Fig 4. Strain-stress curves of group $S$ and group $T$. The yield point and failure point with maximal force are marked.

The ultimate failure loads in group $\mathrm{T}(278.2 \pm 54 \mathrm{~N})$ were significantly greater than those in group S $(249.4 \pm 32 \mathrm{~N})$ $(p=0.028)$. On the contrary, the stiffness values in group $\mathrm{T}(28.5 \pm 4.0 \mathrm{~N} / \mathrm{mm})$ were significantly smaller than those in group $\mathrm{S}(32.3 \pm 4.5 \mathrm{~N})(P=.028)$.

The strain-stress curves of group $S$ and group $\mathrm{T}$ were illustrated in Figure 4. In group S, both the upper and lower yield points existed, and there was a flat curve between lower yield point and the maximal stress point. In group $\mathrm{T}$, only a yield point was found, and the distance between the yield point and the maximal stress point was short. The slope of linear portion in the stress-strain curve up to the yield point represented the stiffness.

In group S, 10 of 12 specimens failed due to the tendon being cut through by the sutures, whereas suture breakage at the tendon-suture interface was found in the remainder of the specimens $(2 / 12)$. In group T, 8 of 12 specimens failed due to a tendon tear, whereas suture breakage occurred in the rest of the specimens (4/12). No anchor pull-out from the humerus was identified in all specimens.

\section{Discussion}

The principal findings of this study indicated that using high-tensile strength tapes in subpectoral biceps tenodesis using a suture anchor leads to significantly greater ultimate failure load but lower levels of stiffness as compared with using high-tensile strength sutures. Open subpectoral biceps tenodesis is popular, ${ }^{9}$ and using suture anchors in open subpectoral biceps tenodesis has been promoted, since some possible complications can be avoided. ${ }^{10,14}$ Among the different suture techniques in subpectoral biceps tenodesis using a suture anchor, the Krackow suture technique has been shown to have superior biomechanical properties. ${ }^{16,17}$ In addition, a high-tensile strength tape has been developed. It features a greater ultimate failure load than the high-tensile strength suture and has the potential to decrease tendon cut through. ${ }^{18}$ Our study thus further evaluated the use of high-tensile strength tapes in subpectoral biceps tenodesis using a suture anchor. Unfortunately, the results of our study did not entirely fit the proposed hypothesis. Despite of this, the difference in the means in stiffness was not large.

Using tape-type sutures has the potential to enhance biomechanical structure in biceps tenodesis using a suture anchor. Ono et al. ${ }^{28}$ indicated that tape-type sutures may be protective in terms of sutures pulling through tendon during loading since tape-type sutures enlarge the suture holes less and displace less than standard no.2 sutures after repetitive tractions. Similarly, Leishman and Chudik reported that a high-tensile strength tape, SutureTape, has better knot security and greater ultimate load to failure than FiberWire, a no. 2 high-tensile strength suture. ${ }^{18}$ Therefore, it was reasonable to expect a significantly greater ultimate failure load in the SutureTape group than in the FiberWire group in the present study.

It is interesting to notice that the group $S$ had significantly greater stiffness values than the group $\mathrm{T}$, although the ultimate failure load in group $\mathrm{T}$ was significantly greater than the group $\mathrm{S}$, and the failure elongation were similar in 2 groups. The aforementioned findings could be explained from the stress-strain curve in 2 groups. In the present study, the stiffness was calculated by the slope of linear portion in the elastic region in the stress-strain curve. Take Figure 4 as an example; a steep slope could be found in the group S, whereas the slope of linear curve in the group $\mathrm{T}$ was relative flat. However, the displacements after yield points were different between the 2 groups. In the group $\mathrm{T}$, the failure point was close to the yield point; in the group $\mathrm{S}$, however, both upper and lower yield points existed, and the curve between yield point and failure point was flat, resulting in greater failure displacement. 
Stiffness is a structural property; the shape of a stiff structure changed only slightly under elastic loads. The present study found significantly greater stiffness values in the $\mathrm{S}$ group as compared with the $\mathrm{T}$ group. The aforementioned finding was unexpected since the previous study reported that tape-type sutures (FiberTape, Arthrex) had significantly greater stiffness values than no. 2 sutures (FiberWire, Arthrex) in a sheep infraspinatus tendon model. ${ }^{28}$

Some possibly factors may play roles in these differences. The first factor was the tendons in small size (approximately $4.5 \mathrm{~mm}$ in width) in this study that were different from the previous study ${ }^{28}$ using tendons $10 \mathrm{~mm}$ in width. As tape-type sutures created larger hole volumes through the tendon than standard No. 2 sutures $\left(3.0 \mathrm{~mm}^{2}\right.$ vs $\left.1.8 \mathrm{~mm}^{2}\right),{ }^{28}$ we thus infer that this larger defect may especially compromise the suture-tendon interface in smaller tendons. Other factors, such as knot security of different suture materials, may also contribute to the difference.

Our biomechanical testing, results were generally consistent with those of previous studies. Hong et al. ${ }^{16}$ conducted a biomechanical study for subpectoral biceps tenodesis and found a mean failure loads of $283.5 \mathrm{~N}$ for the Krackow suture technique group. Despite different sutures obtained from different companies, the mean ultimate failure loads in our study were at similar levels, $257 \mathrm{~N}$ and $295 \mathrm{~N}$ in group $\mathrm{S}$ and group $\mathrm{T}$, respectively. In contrast, Hong et al. ${ }^{16}$ reported tendon being cut through by the sutures as well as suture breakage at the tendon-suture interface as possible failure modes for the Krackow suture technique group. Comparably, both of these types of failure modes were observed in the present study.

\section{Limitations}

There are some limitations in this study. First, the healing process and effects of postoperative rehabilitation could not be fully simulated in this ex vivo timezero biomechanical study. Second, artificial humeri and porcine tendons were used in this study, rather than cadaveric specimens. Despite the different structures, previous studies have suggested that both artificial humeri ${ }^{19,20}$ and porcine tendons ${ }^{22}$ are eligible surrogates for cadaveric specimens. In addition, since the quality of artificial humeri and porcine tendons were controlled, the effects of different suture materials on the fixation strength could be better evaluated than would be the case otherwise. Lastly, the results from this study can only be applied to the assessed technique, the Krackow suture technique.

\section{Conclusions}

Using high-tensile strength tapes in subpectoral biceps tenodesis using a suture anchor leads to significantly greater ultimate failure load as compared with using high-tensile strength sutures in a porcine model. Although lower levels of stiffness were found in hightensile strength tape group, the difference in the means were not large between 2 groups.

\section{Acknowledgments}

We thank Skeleton Materials and Bio-compatibility Core Lab, Research Center of Clinical Medicine, National Cheng Kung University Hospital, Tainan, Taiwan, for assistance with this project.

\section{References}

1. Nho SJ, Strauss EJ, Lenart BA, et al. Long head of the biceps tendinopathy: Diagnosis and management. J Am Acad Orthop Surg 2010;18:645-656.

2. Wu PT, Jou IM, Yang CC, et al. The severity of the long head biceps tendinopathy in patients with chronic rotator cuff tears: Macroscopic versus microscopic results. J Shoulder Elbow Surg 2014;23:1099-1106.

3. Creech MJ, Yeung M, Denkers M, Simunovic N, Athwal GS, Ayeni OR. Surgical indications for long head biceps tenodesis: A systematic review. Knee Surg Sports Traumatol Arthrosc 2016;24:2156-2166.

4. De Carli A, Vadala A, Zanzotto E, et al. Reparable rotator cuff tears with concomitant long-head biceps lesions: Tenotomy or tenotomy/tenodesis? Knee Surg Sports Traumatol Arthrosc 2012;20:2553-2558.

5. Ren YM, Duan YH, Sun YB, Yang T, Hou WY, Tian MQ. Is arthroscopic repair superior to biceps tenotomy and tenodesis for type II SLAP lesions? A meta-analysis of RCTs and observational studies. J Orthop Surg Res 2019;14:48.

6. Zhang Q, Zhou J, Ge H, Cheng B. Tenotomy or tenodesis for long head biceps lesions in shoulders with reparable rotator cuff tears: A prospective randomised trial. Knee Surg Sports Traumatol Arthrosc 2015;23:464-469.

7. Fang JH, Dai XS, Yu XN, et al. Lesions of the long head of the biceps tendon concomitant with rotator cuff tears: Tenotomy or subpectoral mini-open tenodesis? A comparative short to mid-term follow-up study. Orthop Surg 2019;11:857-863.

8. Werner BC, Brockmeier SF, Gwathmey FW. Trends in long head biceps tenodesis. Am J Sports Med 2015;43: 570-578.

9. Corpus KT, Garcia GH, Liu JN, et al. Long head of biceps tendon management: A survey of the American shoulder and elbow surgeons. HSS J 2018;14:34-40.

10. Arena C, Dhawan A. Mini-open subpectoral biceps tenodesis using a suture anchor. Arthrosc Tech 2017;6: e1625-e1631.

11. Beason DP, Shah JP, Duckett JW, Jost PW, Fleisig GS, Cain EL Jr. Torsional fracture of the humerus after subpectoral biceps tenodesis with an interference screw: A biomechanical cadaveric study. Clin Biomech (Bristol, Avon) 2015;30:915-920.

12. Nho SJ, Reiff SN, Verma NN, Slabaugh MA, Mazzocca AD, Romeo AA. Complications associated with subpectoral biceps tenodesis: Low rates of incidence following surgery. J Shoulder Elbow Surg 2010;19:764-768. 
13. Park JS, Kim SH, Jung HJ, Lee YH, Oh JH. A prospective randomized study comparing the interference screw and suture anchor techniques for biceps tenodesis. Am J Sports Med 2017;45:440-448.

14. Frank RM, Bernardoni ED, Veera SS, et al. Biomechanical analysis of all-suture suture anchor fixation compared with conventional suture anchors and interference screws for biceps tenodesis. Arthroscopy 2019;35:1760-1768.

15. Gigi R, Dolkart O, Sharfman ZT, et al. Biomechanical evaluation of two arthroscopic techniques for biceps tenodesis: Triple loop suture versus simple suture. J Shoulder Elbow Surg 2017;26:165-169.

16. Hong CK, Hsu KL, Kuan FC, et al. The Double Krackow suture technique does not offer a significant benefit compared to the Krackow suture technique in subpectoral biceps tenodesis using a double-loaded suture anchor. Arthroscopy 2020;36:701-707.

17. Kaback LA, Gowda AL, Paller D, Green A, Blaine T. Long head biceps tenodesis with a knotless cinch suture anchor: A biomechanical analysis. Arthroscopy 2015;31:831-835.

18. Leishman DJ, Chudik SC. Suture tape with broad full-width core versus traditional round suture with round core: A mechanical comparison. Arthroscopy 2019;35:2461-2466.

19. Elfar J, Menorca RM, Reed JD, Stanbury S. Composite bone models in orthopaedic surgery research and education. J Am Acad Orthop Surg 2014;22:111-120.

20. Grover P, Albert C, Wang M, Harris GF. Mechanical characterization of fourth generation composite humerus. Proc Inst Mech Eng H 2011;225:1169-1176.

21. Golish SR, Caldwell PE 3rd, Miller MD, et al. Interference screw versus suture anchor fixation for subpectoral tenodesis of the proximal biceps tendon: A cadaveric study. Arthroscopy 2008;24:1103-1108.

22. Domnick C, Wieskotter B, Raschke MJ, et al. Evaluation of biomechanical properties: Are porcine flexor tendons and bovine extensor tendons eligible surrogates for human tendons in in vitro studies? Arch Orthop Trauma Surg 2016;136:1465-1471.

23. Mirzayan $\mathrm{R}$, Takara $\mathrm{T}$, Batech $\mathrm{M}$, McCrum CL. In vivo analysis of biceps tendon characteristics in subpectoral tenodesis. Arthroscopy 2017;33:1495-1502.

24. Chiang FL, Hong CK, Chang CH, Lin CL, Jou IM, Su WR. Biomechanical comparison of all-suture anchor fixation and interference screw technique for subpectoral biceps tenodesis. Arthroscopy 2016;32:1247-1252.

25. Hong CK, Chang CH, Chiang FL, et al. Biomechanical properties of suprapectoral biceps tenodesis: Double knotless screw fixation is superior to single knotless screw fixation. Arch Orthop Trauma Surg 2018;138:1127-1134.

26. Hong CK, Hsu KL, Kuan FC, Lin CL, Yeh ML, Su WR. Biomechanical evaluation of a transtendinous all-suture anchor technique versus interference screw technique for suprapectoral biceps tenodesis in a cadaveric model. Arthroscopy 2018;34:1755-1761.

27. Su WR, Budoff JE, Chiang CH, Lee CJ, Lin CL. Biomechanical study comparing biceps wedge tenodesis with other proximal long head of the biceps tenodesis techniques. Arthroscopy 2013;29:1498-1505.

28. Ono Y, Joly DA, Thornton GM, Lo IKY. Mechanical and imaging evaluation of the effect of sutures on tendons: Tape sutures are protective to suture pulling through tendon. J Shoulder Elbow Surg 2018;27:1705-1710. 